\title{
Cognitive Function, Emotional and Behavioral Problems, and Temperament of Premature Children
}

\author{
Dong-hyun Ahn',2, Aran Min ${ }^{1}$, Kangryul Kim¹, Kyung-ah Kim¹, Mi-Young Oh${ }^{1}$, \\ Hyun Ju Lee ${ }^{3}$, Hyun-Kyung Park ${ }^{3}$, and Hyewon Park ${ }^{4}$ \\ ${ }^{1}$ Department of Psychiatry, Hanyang University Hospital, Seoul, Korea \\ ${ }^{2}$ Department of Psychiatry, Hanyang University College of Medicine, Seoul, Korea \\ ${ }^{3}$ Department of Pediatrics, Hanyang University College of Medicine, Seoul, Korea \\ ${ }^{4}$ Department of Child \& Family Welfare, University of Ulsan, Ulsan, Korea
}

\begin{abstract}
Objectives: We aimed to compare preterm, neurodevelopmentally disordered and healthy full-term children.
Methods: We enrolled 47 children who were born preterm, 40 neurodevelopmentally disordered children, and 80 healthy children as control participants, in order to assess the cognitive functioning and the risk of behavioral problems at the age of 5 . Children were assessed using the Korean Wechsler Preschool and Primary Scale of Intelligence-4th edition (K-WPPSI-IV), the Child Behavior Checklist (CBCL), and the Temperament and Character Inventory (TCI).

Results: The mean K-WPPSI-IV score of the preterm group was $87.19 \pm 17.36$, which was significantly higher than that of the neurodevelopmental disorder group $(69.98 \pm 28.63 ; \mathrm{p}<0.001)$ but lower than that of the control group $(107.74 \pm 14.21 ; \mathrm{p}<0.001)$. The cumulative CBCL scores of the preterm children were not significantly different from those of the control group. Additionally, the TCI scores for reward dependence of the preterm children were higher than those of the control group.

Conclusion: The cognitive performance of preterm infants was lower than that of healthy full-term infants at the age of 5, and there was an association between slower growth and decreased cognitive ability.
\end{abstract}

Key Words: Preterm; Korean Wechsler Preschool and Primary Scale of Intelligence; Cognitive function; Emotional and behavioral problems; Temperament.

Received: August 7, 2018 / Revision: October 2, 2018 / Accepted: October 16, 2018

Address for correspondence: Dong-hyun Ahn, Department of Psychiatry, Hanyang University Hospital, 222 Wangsimni-ro, Seongdong-gu, Seoul 04763, Korea

Tel: +82-2-2290-8425, Fax: +82-2-2298-2055, E-mail: ahndh@hanyang.ac.kr

\section{INTRODUCTION}

The expression "preterm babies" refers to newborns born before 37 weeks of gestation or before 259 days from final menstruation. Depending on the length of the gestation, they can be subdivided into Late Preterm (between 32 and 37 weeks), Very Preterm (between 28 and 32 weeks), and Extremely Preterm (less than 28 weeks) [1]. As the fetal brain develops during pregnancy, preterm babies are predicted to have lower levels of cognitive functions in comparison to fullterm babies.

This assumption has been supported in a number of clinical experiments on preterm babies. A large-scale research conducted in Taiwan evaluated the intelligence of 3225 -yearolds who were born as very low-weight infants and 1035 -yearolds who were born as full-term infants using the Wechsler

This is an Open Access article distributed under the terms of the Creative Commons Attribution Non-Commercial License (https://creativecommons.org/licenses/by-nc/4.0) which permits unrestricted non-commercial use, distribution, and reproduction in any medium, provided the original work is properly cited.
Preschool and Primary Scale of Intelligence-Revised (WPPSI-R) [2]. This study showed that full-term children had a mean score of $114.9 \pm 11.8$ on the Performance Scale, $103.9 \pm 12.9$ on the Verbal Scale, and $110.6 \pm 11.2$ on the Full Scale, while very low-weight children showed mean scores of $94.1 \pm 16.4$ on the Performance Scale, $87.2 \pm 12.8$ on the Verbal Scale, and $89.5 \pm 14.6$ on the Full Scale, indicating that very lowweight children have worse cognitive functions in comparison to full-term children. Another study based on the WPPSI-R evaluation of 6-year olds showed that children born as full-term infants scored $106.2 \pm 14.3$ on the Verbal Scale, which was significantly different from the average of $95.3 \pm 11.7 \mathrm{ex}-$ hibited by 24 children who were born as very low-weight infants [3]. Another study showed that the average cognitive function test score was significantly related to birth weight, regardless of the age at which the test was performed [4]. Therefore, the decreased cognitive function of preterm infants has been reported in previous studies.

The recently revised WPPSI-IV is suggested to be especial- 
ly useful to evaluate infants with various neurodevelopmental disorders, because many studies show its relationship with neuroscientific opinions about neurodevelopment and cognitive functions.

Furthermore, there have been some studies about the emotional behaviors of preterm infants. In a study of the association of in-class behaviors and birth weight of elementary school children, children with lower birth weight showed worse attention and verbal skills [5]. In comparison with normal children and low-weight children, very low-weight children showed significantly greater difficulty adapting to school and more hyperactivity [6]. A meta-analysis of 6 previous studies compiled by Bhutta et al. [4] reported the preterm children's relative risk factor for attention-deficit/hyperactivity disorder (ADHD) to be 2.64. Other studies are available about the behavioral problems of preterm or very low-weight children.

Some studies are also available on the temperament of preterm infants. Sajaniemi et al. [7] compared preterm and fullterm infants using the Toddler Temperament Questionnaire. The distribution of temperament type (easy, difficult, slow to warm up) was not significantly related to birth weight, but preterm infants showed lower activity, more adaptability, more positive mood, lower intensity emotions, and lower reaction threshold. Another study showed that preterm toddlers were more likely to have easier temperaments and less likely to have difficult temperaments, but there were no significant differences in temperament and behavior depending on the time of birth [8]. However, there has been no previous study on the temperament of preterm infants using Cloninger's seven-factor model, which can predict personality temperaments.

The Diagnostic and Statistical Manual of Mental Disorders, 5th edition (DSM-5), recently revised by the American Psychiatric Association suggests a new scope for the diagnosis of neurodevelopmental disorders, in which intellectual developmental disorder, autism spectrum disorder, ADHD, communication disorder, motor disorder, and specific learning disorder are included. The common feature among all these is that the symptoms generally occur in the early developmental stages, and that lack of development leads to impairment in personal, social, academic, or occupational functions. Preterm birth is known to be one of the risk factors of developing these neurodevelopmental disorders.

Although many studies have shown evidence that preterm children have worse cognitive functions in comparison to normal children, there is a lack of research on the comparison to children with neurodevelopmental disorders. In the case of children with neurodevelopmental disorders, the degree of cognitive function may be varied, but it can be predicted that cognitive functions of children with neurodevelopmen- tal disorders, serious enough to receive outpatient treatment, will be worse than those of normal children. Therefore, we formulated the hypothesis that the cognitive functions of preterm children will be worse than those of normal children, but better than those of children with neurodevelopmental disorders, and designed our research to test this hypothesis.

In our study, we aimed to examine the clinical meaning of the cognitive impairment and behavioral problems of preterm children by comparing preterm children, children with neurodevelopmental disorders, and normal children in order to study the relationship among brain development, cognitive impairment, and behavioral problems. Furthermore, we also conducted an evaluation of children's temperament, based on the seven-factor model, to examine whether the behavioral problems of preterm children arise from their temperament or are acquired from the environment.

\section{METHODS}

\section{Subjects}

Preterm children, children with neurodevelopmental disorders, and normal children between the ages of 2 years and 6 months and 7 years and 7 months were enrolled in the study. Preterm children $(n=47)$ were recruited, via phone calls and by visiting the Department of Pediatrics, among children who were under 32 weeks of gestation at birth, with a history of being admitted to a University Hospital located in Seoul. Children with neurodevelopmental disorders $(n=40)$ were recruited, via phone calls and visiting the Department of Psychiatry, among children in the desired age group who were receiving outpatient treatment based on DSM diagnostic criteria in the University Hospital. Normal children $(\mathrm{n}=80)$ were recruited via convenience sampling among children in the desired age group who attended regular daycare centers, kindergarten, and elementary schools in metropolitan Seoul, and voluntarily expressed the desire to participate, with the approval of their parents. However, children who were confirmed to have neurodevelopmental disorders or those who were suspected of have clinical problems based on the Korean version of the Child Behavior Checklist (K-CB$\mathrm{CL}$ ) were excluded from the control group. The division between younger-age and older-age groups is shown in Table 1.

\section{Evaluation tools}

Korean-Wechsler Preschool and Primary Scale of Intelligence-4th edition (K-WPPSI-IV)

The K-WPPSI-IV was developed in the United States in 2012 and translated and standardized into Korean by Park et al. 
Table 1. Age distribution of group of subjects

\begin{tabular}{lccc}
\hline & \multicolumn{2}{c}{ Age } & \\
\cline { 2 - 3 } & $\begin{array}{c}\text { Younger ones } \\
(2: 6-3: 11)\end{array}$ & $\begin{array}{c}\text { Older ones } \\
(4: 0-7: 7)\end{array}$ & Total \\
\hline Group & & & \\
Preterm & 23 & 24 & 47 \\
NDD & 5 & 35 & 40 \\
Control & 29 & 51 & 80 \\
Total & 57 & 110 & 167 \\
\hline
\end{tabular}

NDD: neurodevelopmental disorder

$[9,10]$. The K-WPPSI-IV is intended for young children, between the ages of 2 years and 6 months and 7 years and 7 months. It comprises the Full Scale Intelligence Quotient (FSIQ), which represents overall cognitive skills, index scores representing the cognitive skills for specific cognitive areas (Verbal Comprehension, Visual Spatial, Fluid Reasoning, Working Memory, Processing Speed), and 15 subtest scores. To reduce the burden of conducting all the subtests on young children, the evaluation system and records were divided into those appropriate for children between 2 years and 6 months and 3 years and 11 months, and those appropriate for children between 4 years and 0 months and 7 years and 7 months. The average reliability of the K-WPPSI-IV was shown to be given by a Cronbach's $\alpha$ of 0.92 .

\section{Korean version of the Child Behavior Checklist for ages} 1.5-5 (K-CBCL 1.5-5)

Achenbach and Rescorla [11] developed the CBCL 1.5-5 and Kim et al. [12] translated and standardized it into Korean version. Ninety-nine items out of 100 are scored 0 , 1 , or 2 points, and 1 item is open-ended and used to describe the problems of young children and evaluate their degree. The main caretaker considers the frequency of behaviors in the past 2 months to evaluate 7 subareas, which are emotional responsiveness, anxious/depressed, somatic complaints, withdrawn, sleep problems, attention problems, and aggressive behavior, as well as 2 factor scores of internalizing and externalizing problems, and a cumulative behavioral problem score. The range of internal consistency and reliability of the K-CBCL 1.5-5 between syndrome indices and internalizing and externalizing total score was $0.56-0.94$ in terms of Cronbach's $\alpha$, while the Cronbach's $\alpha$ for the total score including all items was 0.94 , and the same value was obtained for internalizing problems and externalizing problems.

\section{The Korean Version of the Preschool Temperament and Character Inventory (K-psTCI)}

Cloninger's Temperament and Character Inventory (TCI), which was originally a self-reported survey format [13], was adapted by Constantino et al. [14] to use on children of preschool ages and further translated and standardized into Korean by Park et al. [15]. The 4 factors of temperament are Novelty Seeking (NS), Harm Avoidance (HA), Reward Dependence (RD), and Persistence (P), and the 3 factors of personality are Self-Directedness, Cooperativeness, and SelfTranscendence. A total of 74 items include 40 items on temperament and 34 items on personality. Each item on the 4 temperament factors are scored on a scale of $1-5$, ranging from 'strongly disagree' to 'strongly agree.' 32 items randomly mixed in within the 74 items have a reversed 5-point scale. The internal consistency and reliability range of K-preschool TCI was 0.62-0.78 (Cronbach's $\alpha$ ).

\section{Process}

This research was approved by the Institutional Review Board of the University Hospital in which this study was conducted (IRB No. 2013-12-001). Parents of the volunteer subjects were informed of the research background and purpose, process, risk and discomfort due to participation, and benefits, after which consent was received and the evaluation conducted. The total evaluation time was between 40 minutes and 2 hours and 40 minutes, with large variance depending on the child's cognitive and cooperation levels. The evaluation was carried out in the following order: examination instructions and agreement, examination of the child's intelligence, self-reported exam of the mother, and interview about special factors. The intelligence examination was conducted by 3 clinical psychology trainees, who are training with clinical psychologists at a University Hospital after graduating with a master's degree in a psychology-related field, using standardized procedures. The examiners completed a 3-sessions workshop about conducting, scoring, and coding KWPPSI-IV and were trained on the standardized procedures. After completing the examination, the parents of the child were given free feedback about the results of the research examination, including the level of cognitive function development.

\section{Statistical analysis}

Data were processed and analyzed using SPSS 18.0 (SPSS Inc., Chicago, IL, USA) by statistician in University Medical Research Assist-Center. To examine the sociodemographic characteristics, the chi squared and analysis of variance (ANOVA) tests were used. The intelligence index data for each group did not fit the equal variance assumption according to Levene's test, so Welch's ANOVA was used, and the GamesHowell test was used for post-hoc analysis. Since, instead, the data on behavioral problems and temperament fit the equal variance assumption, ANOVA was used, with Scheffe's meth- 
od for post-hoc analysis.

Given that the sub-indexes for the 2 years and 6 months to 3 years and 11 months, and from 4 years and 0 months to 7 years and 7 months age groups differ in the WPPSI-IV, the subjects were divided into a younger-age and an older-age group for statistical analysis. The common subtests were analyzed using data from all subjects, but fluid reasoning and processing speed, which are only evaluated in the older-age group, were only analyzed for this group.

The sub-indexes for the from 1 years and 6 months to 5 years and 11 months, and from 6 years and 0 months to 7 years and 7 months age groups are different for the CBCL as well, so only the common indexes were analyzed for statistical analysis.

\section{RESULTS}

\section{Sociodemographic characteristics}

The sociodemographic background of the research sub- jects is shown in Table 2. Gender, age, birth order, period of gestation at birth, mother's age at pregnancy, plan of pregnancy, marriage status, number of family members, number of children, education level of father and mother were investigated and subjected to post-hoc analysis for the three groups. Age and period of gestation at birth showed statistically significant differences. Children in the neurodevelopmental disorder group were older than those in the preterm and control groups. The period of gestation at birth was significantly lower for preterm group, which is expected given the fact that such group consists of children whose gestation period was 37 weeks or shorter.

\section{Cognitive function}

The results of the K-WPPSI-IV cognitive exam are shown in Table 3. The FSIQ, Verbal Comprehension Index (VCI), Visual Spatial Index (VSI), Fluid Reasoning Index (FRI), Working Memory Index (WMI), and Processing Speed Index (PSI) all showed significant differences among the three

Table 2. Sociodemographic characteristics of subjects

\begin{tabular}{|c|c|c|c|c|c|}
\hline Variables & Preterm' $(n=47)$ & $\operatorname{NDD}^{2}(n=40)$ & Control $^{3}(n=80)$ & $F$ & Post hoc \\
\hline Age, years & $3.94(1.47)$ & $5.27(1.31)$ & $4.30(1.39)$ & $1.412^{*}$ & $2>1,3$ \\
\hline Sex & & & & 2.998 & \\
\hline Male & 22 & 27 & 42 & & \\
\hline Female & 25 & 14 & 38 & & \\
\hline Gestational period & $28.31(3.52)$ & $37.62(5.38)$ & $39.09(1.41)$ & $54.405^{*}$ & $2,3>1$ \\
\hline Age of pregnancy & $32.56(4.43)$ & $31.87(4.08)$ & $31.60(3.53)$ & 0.849 & \\
\hline Scheduled pregnancy & & & & 2.196 & \\
\hline Yes & 33 & 23 & 50 & & \\
\hline No & 12 & 16 & 30 & & \\
\hline Status of parents & & & & 8.706 & \\
\hline Marriage & 44 & 33 & 74 & & \\
\hline Divorced & 1 & 6 & 3 & & \\
\hline Separated & 0 & 1 & 2 & & \\
\hline Number of family & $4.44(1.26)$ & $4.15(0.92)$ & $4.20(0.97)$ & 0.854 & \\
\hline Number of children & $1.82(0.68)$ & $1.83(0.64)$ & $1.94(0.71)$ & 0.588 & \\
\hline Birth ranking & & & & 4.784 & \\
\hline 1st & 25 & 26 & 35 & & \\
\hline 2nd & 15 & 11 & 35 & & \\
\hline 3rd and more & 3 & 2 & 6 & & \\
\hline Educational levels of father & & & & 5.157 & \\
\hline High school & 9 & 10 & 21 & & \\
\hline University & 26 & 19 & 38 & & \\
\hline Graduate & 8 & 6 & 13 & & \\
\hline Etc & 0 & 1 & 0 & & \\
\hline Educational levels of mother & & & & 12.439 & \\
\hline High school & 14 & 9 & 29 & & \\
\hline University & 24 & 18 & 37 & & \\
\hline Graduate & 5 & 9 & 8 & & \\
\hline Etc & 0 & 2 & 0 & & \\
\hline
\end{tabular}

Data are mean (standard deviation) values. ${ }^{*} p<0.001$. Ełc: et cetera, NDD: neurodevelopmental disorder 
groups. The post-hoc analysis showed that the average cognitive indexes for all areas in the preterm group were lower in comparison to the control group and higher in comparison to the neurodevelopmental disorder group.

\section{Emotional \& behavioral problems}

The results about total score, internalizing problems, and externalizing problems extracted from the CBCL are shown in Table 4. The result of the CBCL evaluation of the three groups showed significant differences among them in the total score, internalizing problems index, and externalizing problems index. The Bonferroni post-hoc test among the three groups showed that the neurodevelopmental disorder group showed significantly higher behavioral problem scores compared to the preterm and control groups, but there was no difference between the preterm and control groups.

The comparisons of the subareas of the CBCL are shown in Table 5. The analysis of the subareas common to all age groups showed significant differences among the three groups in the anxiety/depression index, attention index, and aggressive behavior index. The anxiety/depression index was significantly higher in the neurodevelopmental disorder group than in the control group. The neurodevelopmental disorder group showed significantly higher attention and aggressive behavior scores than the preterm and control groups, but there was no significant difference between the preterm and control groups. There was no significant difference among the groups in the somatic complaints index.

Table 3. Comparison of results of WPPSI-IV between preterm children group, NDD children group, and control group

\begin{tabular}{|c|c|c|c|c|c|}
\hline Subtest & Preterm' $(n=47)$ & $\mathrm{NDD}^{2}(\mathrm{n}=40)$ & Control $^{3}(\mathrm{n}=80)$ & $\mathrm{F}$ & Post hoc \\
\hline FSIQ & $87.19(17.36)$ & $69.98(28.63)$ & $107.74(14.21)$ & $46.10^{*}$ & $2<1<3$ \\
\hline $\mathrm{VCl}$ & $92.49(16.95)$ & $74.12(29.72)$ & 112.39 (13.36) & $46.21^{*}$ & $2<1<3$ \\
\hline VSI & $87.28(16.86)$ & $77.45(26.48)$ & $103.44(14.06)$ & $26.86^{*}$ & $2<1<3$ \\
\hline WMI & 95.89 (17.90) & $74.63(25.35)$ & $106.24(14.35)$ & $28.53^{*}$ & $2<1<3$ \\
\hline FRI & 91.13 (16.30) & $74.77(26.86)$ & $104.35(16.16)$ & $18.56^{*}$ & $2<1<3$ \\
\hline PSI & 87.00 (19.03) & $70.23(23.96)$ & $105.06(15.01)$ & $31.79 *$ & $2<1<3$ \\
\hline
\end{tabular}

Post hoc Games-Howell test. Data are mean (standard deviation) values. *p<0.001. FRI: Fluid Reasoning Index, FSIQ: Full Scale Intelligence Quotient, NDD: neurodevelopmental disorder, PSI: Processing Speed Index, VCI: Verbal Comprehension Index, VSI: Visual Spatial Index, WMI: Working Memory Index, WPPSI-IV: Wechsler Preschool and Primary Scale of Intelligence-4th edition

Table 4. The comparison of CBCL ratings among preterm, NDD, and control groups

\begin{tabular}{|c|c|c|c|c|c|}
\hline & Preterm ${ }^{1}(\mathrm{n}=47)$ & $\operatorname{NDD}^{2}(n=40)$ & Control $^{3}(\mathrm{n}=80)$ & $\mathrm{F}$ & Post hoc \\
\hline Total score & $50.42(1.01)$ & $62.46(8.61)$ & $49.77(1.48)$ & $23.23^{*}$ & $2>1,3$ \\
\hline Internalizing problems & $49.64(9.22)$ & $58.36(7.42)$ & $49.67(9.82)$ & $13.39 *$ & $2>1,3$ \\
\hline Externalizing problems & $5.42(1.61)$ & $62.28(9.51)$ & $5.77(1.24)$ & $19.28 *$ & $2>1,3$ \\
\hline
\end{tabular}

Post hoc Scheffe test. Data are mean (standard deviation) values. * $\mathrm{p}<0.001$. CBCL: Child Behavior Checklist for Ages 1.5-5, NDD: neurodevelopmental disorder

Table 5. Comparison of result of CBCL subtests between preterm children group, NDD children group and control group

\begin{tabular}{|c|c|c|c|c|c|}
\hline Subtest & Preterm $^{1}(n=47)$ & $\operatorname{NDD}^{2}(n=40)$ & Control $^{3}(n=80)$ & $\mathrm{F}$ & Post hoc \\
\hline Anxious/depressed & $53.73(5.61)$ & $57.74(7.62)$ & $51.91(9.99)$ & $6.23^{*}$ & $2>3$ \\
\hline Somatic complaints & $54.00(5.57)$ & $53.85(5.48)$ & $51.86(9.86)$ & 1.378 & \\
\hline Attention problems & $54.51(6.03)$ & $63.03(9.38)$ & $52.16(1.23)$ & $19.04^{\dagger}$ & $2>1,3$ \\
\hline Aggressive behavior & $54.11(6.89)$ & $61.03(8.95)$ & $52.36(12.19)$ & $9.55^{\dagger}$ & $2>1,3$ \\
\hline
\end{tabular}

Post hoc Scheffe test. Data are mean (standard deviation) values. ${ }^{*} p<0.01,{ }^{\dagger} p<0.001 . \mathrm{CBCL}$ : Child Behavior Checklist for Ages 1.5-5, NDD: neurodevelopmental disorder

Table 6. Comparison of result of TCl between preterm children group, NDD children group, and control group

\begin{tabular}{|c|c|c|c|c|c|}
\hline Subtest & Preterm' $(n=47)$ & $\operatorname{NDD}^{2}(n=40)$ & Control $^{3}(n=80)$ & $\mathrm{F}$ & Post hoc \\
\hline Novelty seeking & $49.13(11.22)$ & $57.10(1.74)$ & $51.34(9.04)$ & $6.68^{*}$ & $2>1,3$ \\
\hline Harm avoidance & $49.69(7.52)$ & $54.10(11.17)$ & $50.89(9.47)$ & 2.31 & \\
\hline Reward dependence & $52.72(11.53)$ & $47.36(12.20)$ & $47.68(8.31)$ & $3.63^{*}$ & $1>3$ \\
\hline Persistence & $48.36(11.90)$ & $41.23(1.09)$ & $48.30(9.34)$ & $6.93^{\dagger}$ & $2<1,3$ \\
\hline
\end{tabular}

Post hoc Scheffe test. Data are mean (standard deviation) values. ${ }^{*} p<0.01,{ }^{\dagger} p<0.001$. NDD: neurodevelopmental disorder, TCl: Temperament and Character Inventory 


\section{Infant temperament}

The evaluation results for the temperament and personality of the three groups are shown in Table 6. Regarding temperament factors, the NS, RD, and P indexes showed significant differences among the three groups. The NS score was significantly higher in the neurodevelopmental disorder group than in the preterm and control groups, while the difference between preterm group and control group was not significant. There was a significant difference between preterm group and control group in the RD index. The $\mathrm{P}$ score was significantly lower for the neurodevelopmental disorder group than for the preterm and control groups, but the difference between the preterm group and the control group was not significant.

\section{DISCUSSION}

There were significant differences among the three groups on the intelligence index of all subtests, including the FSIQ. The control group showed the highest intelligence, followed by the preterm group, while the neurodevelopmental disorder group was evaluated to be the lowest. In a previous study using the WPPSI-R tool to compare preterm group to the control group, the preterm group scored lower than the control group on the full scale, verbal scale, and performance scale of intelligence, with differences in mean scores of about 10$20[2,3,16,17]$. Similarly, comparing the preterm group with K-WPPSI-IV in our study also showed differences of about $10-20$ points among the neurodevelopmental disorder, preterm, and control groups in the full scale intelligence, which aligns with previous intelligence evaluation results. In our study we compared the neurodevelopmental disorder group to the preterm group, and we found that the preterm group showed higher score than the neurodevelopmental disorder group by about $10-20$ points. Even when considering the possible inclusion of children with intellectual disability, who can affect the average, our results show clinically that the preterm group experiences a cognitive function impairment that is not as severe as the neurodevelopmental disorder group, but still significant compared to the control group. The preterm group shows a lower score than the control group not only in full-scale intelligence, but also in all sub-indexes. In particular, verbal comprehension shows the biggest difference between the preterm and control groups. Considering that the control group's verbal score was measured to be rather high, at 112.39, our result aligns with previous results showing high verbal scores for Korean children [18]. Additionally, Lee et al. [19] found a significant effect of birthweight on verbal comprehension skills, and that a better verbal comprehension skill was associated with higher birth weight regardless of age; considering the fact that preterm infants have lower weight than normal infants, this also aligns with previous research.

Considering the reasons why premature babies have worse cognitive functions, we can assume that since the brain of the fetus is developing during pregnancy, the brain of a premature baby is less fully developed, and therefore exhibits worse cognitive functions, in comparison to a full-term baby. More specifically, the existing literature shows that the growth and migration of brain cells begins at 10 weeks of gestation and ends at 22-24 weeks. When the movement is finished, cortical tissues start to form, and synapses are actively formed until up to 40 weeks [20]. In particular, it can be inferred that wide-ranging cerebral cortex development is difficult in premature infants considering that 100 million synapses form per minute in the last trimester of pregnancy.

Typically, subcortical neurons play a key role in the formation of thalamus-cortex and cortex-cortex synapses. These subcortical neurons are susceptible to hypoxia, and thus immature lungs create problems in the formation of the cortex. In fact, a study confirmed the decrease in academic cognitive function of premature children with bronchial and lung abnormalities [21]. Furthermore, the lack of white matter due to premature birth secondarily affects the development of the cortical region, as it affects the axial connections. Therefore, cognitive skills are expected to decrease for premature babies, consistently with the results of this study.

Evaluation of the emotions and behavior of preterm babies showed that behavioral problems were more frequent and significant in the neurodevelopment disorder group than in the preterm and control groups. Reports by parents of behavioral problems, including internalizing and externalizing problems, in the preterm group were not higher than in the control group, and also showed no consistent results in previous studies. The study by Brown et al. [22] on the CBCL evaluation of 4- to 5-year old preterm children showed them all to be within the normal range. The study by Pomella et al. [23] on the CBCL evaluation of preterm children in all school ages, with comparison to a control group, also showed no clinically significant difference. In contrast, some studies showed increased hyperactivity, attention problems, and incidence of ADHD in preterm children [4-6]. The results of our study show that the preterm group did not have more attentional problems than the control or the neurodevelopmental disorder group. Considering that $\mathrm{ADHD}$ is a very common disorder, occurring in about $5 \%$ of all children, it is difficult to conclude that preterm is a risk factor, even if ADHD symptoms may be observed in preterm children. Various research designs and analysis methods produced inconsistent results about whether ADHD risk is increased in preterm children. Considering that there are differences among examiners in 
evaluating a child's externalizing problems in general, studies based on a consistent evaluation by an objective examiner and objective test data as well as the mothers' evaluation can help mitigate this controversy.

Existing studies on the temperaments of preterm children did not produce consistent results. However, looking at past studies, which showed results similar to those of this study, a higher tendency towards avoidance of new stimuli [24], higher response intensity to external stimuli, and lower level of concentration on the stimuli [25] were reported in preterm children compared to full-term children. In contrast, our study showed that the neurodevelopmental disorder group showed higher NS than the preterm and control groups. Thus, the preterm group did not seek new and rapid stimulation. Although direct comparison is difficult, because existing studies do not use Cloninger's 7-factor model, our results do not confirm the tendency to avoid new stimuli reported in previous studies. This discrepancy may have been affected by differences in variables such as the timing of the assessment and evaluation tools, so further research is expected to be necessary in the future. Moreover, Persistence (P) was lower in the neurodevelopmental disorder group than in the preterm and control groups. This also implies that the preterm group is not particularly less patient. As these findings of our research differ from previous studies, further studies will be needed in the future.

However, RD was significantly higher in the preterm group than in the control group. Temperament is inherited from the parents, but the expression of this temperament and the formation of personality are affected by the development process. Reward dependence is the sensitivity to social reward, which is related to the emotional warmth of the parents. Cloninger's theory suggests that individuals high in reward dependence and low in norepinephrine levels are ambitious, warm, sentimental, sociable, and sensitive, quickly recognize social and unspoken demands, and show effective communication [26-29]. The vulnerability of the preterm child's physical condition increases the awareness and responsiveness of the parents at the beginning of child care. This affects parent-child attachment relationships, and it can be inferred that active material and emotional support by the parents for their preterm children has to do with the increase in reward dependence-that is, responsiveness to others and increased sociability-in the children.

There are a number of limitations in our study. First, since the evaluation tools other than intelligence tests were based on self-reported surveys that parents completed about their children, the subjective perspective of the parents is reflected, and some answers may have been downplayed or exaggerated. Second, due to the cross-sectional nature of this study, only associations among cognitive functions, behavioral problems, personalities and temperaments could be drawn, and we cannot find causal relationships among the results. Third, our subjects were children who visited a hospital in a specific region, which may not be representative of the general demographic characteristics: Thus it is difficult to generalize the results to all Korean children, and to say that our results represent preterm and neurodevelopmentally disordered children in general. Also, it is difficult to say that our control subjects reflect the general demographic characteristics, as we only targeted children living in Seoul. Third, the proportion of younger-age group and older-age group in the preterm group, neurodevelopmental disorder group, and control group were different, and we could not rule out the possibility that such differences affected the results. Furthermore, the neurodevelopmental disorder group in our study also included children with various disorders such as intellectual disorder, autism spectrum disorder, and ADHD. Therefore, our study is limited by the lack of specificity of the neurodevelopmental disorders affecting the children, and thus further research on a more uniform group is needed.

\section{CONCLUSION}

Preterm birth has been known to be a risk factor not only for neurodevelopmental disorders, but also for later emotional and behavioral problems. Comparing preterm children to children with neurodevelopmental disorders and normal children showed that the cognitive functions of the preterm group were higher than those of the neurodevelopmental disorder group, but lower than those of the control group. All the sub-indexes showed the same results, especially in the VCI. There is a lack of agreement on whether preterm children will be troubled in life by a lot of emotional and behavior problems. Our study found no noticeable difference compared to normal children. However, the temperamental distinctive feature of the preterm group was that they showed more reward dependence than did the control group, showing that preterm children are more sensitive to social stimuli than normal children.

\section{Acknowledgments}

This study was funded by the Government (Department of Education) in 2014 through the National Research Foundation of Korea (NRF-2014S1A5A2A01013621) and was financially supported by research funds of Inpsyt.

\section{Conflicts of Interest}

The authors have no financial conflicts of interest 


\section{REFERENCES}

1) Anonymous. WHO: recommended definitions, terminology and format for statistical tables related to the perinatal period and use of a new certificate for cause of perinatal deaths. Modifications recommended by FIGO as amended October 14, 1976. Acta Obstet Gynecol Scand 1977;56:247-253.

2) Wang PW, Fang LJ, Tsou KI. The growth of very-low-birth-weight infants at 5 years old in Taiwan. Pediatr Neonatol 2014;55:114-119.

3) Litt R, Joseph A, Gale R. Six year neurodevelopmental follow-up of very low birthweight children. Isr J Med Sci 1995;31:303-308.

4) Bhutta AT, Cleves MA, Casey PH, Cradock MM, Anand KJ. Cognitive and behavioral outcomes of school-aged children who were born preterm: a meta-analysis. JAMA 2002;288:728-737.

5) Klebanov PK, Brooks-Gunn J, McCormick MC. Classroom behavior of very low birth weight elementary school children. Pediatrics 1994; $94: 700-708$

6) McCormick MC, Gortmaker SL, Sobol AM. Very low birth weight children: behavior problems and school difficulty in a national sample. J Pediatr 1990;117:687-693.

7) Sajaniemi N, Salokorpi T, von Wendt L. Temperament profiles and their role in neurodevelopmental assessed preterm children at two years of age. Eur Child Adolesc Psychiatry 1998;7:145-152.

8) Oberklaid F, Sewell J, Sanson A, Prior M. Temperament and behavior of preterm infants: a six-year follow-up. Pediatrics 1991;87:854861.

9) Wechsler D. Technical and interpretative manual: WPPSI-IV. New York: Pearson Inc;2012.

10) Park H, Seo Y, Lee J. A study of concurrent validities of K-WPPSIIV. Korean J Child Stud 2015;36:65-83.

11) Achenbach TM, Rescorla LA. Manual for ASEBA preschool forms and profiles. Burlington: University of Vermont, Research Center for Children, Youth \& Families;2000.

12) Kim YA, Lee J, Moon SJ, Kim YJ, Oh KJ. Standardization study for the Korean version of the child behavior checklist for ages 1.55. Kor J Clin Psychol 2009;28:117-136.

13) Cloninger CR. A unified biosocial theory of personality and its role in the development of anxiety states. Psychiatr Dev 1986;4:167-226.

14) Constantino JN, Cloninger CR, Clarke AR, Hashemi B, Przybeck T. Application of the seven-factor model of personality to early childhood. Psychiatry Res 2002;109:229-243.

15) Park JK, Kim JW, Ki SW, Shin YJ. Reliability and validity of the Korean version of the preschool temperament and character inventory (K-psTCI). J Korean Neuropsychiatr Assoc 2004;43:425-434.

16) Espírito Santo JL, Portuguez MW, Nunes ML. Cognitive and be- havioral status of low birth weight preterm children raised in a developing country at preschool age. J Pediatr (Rio J) 2009;85:35-41.

17) Meio MD, Lopes CS, Morsch DS, Monteiro AP, Rocha SB, Borges RA, et al. Pre-school cognitive development of very low birth weight preterm children. J Pediatr (Rio J) 2004;80:495-502.

18) Park HW, Lee KO. Intellectual characteristics of Korean children: analyses of K-WPPSI-IV performance. Korean J Child Stud 2016;37: 157-168.

19) Lee SY, Min A, Lee HJ, Park H, Oh MY, Cho JH, et al. The effect of low birth weight and age on the cognitive performance of preterm preschoolers. J Korean Acad Child Adolesc Psychiatry 2017;28:141148.

20) Nosarti C, Murray RM, Hack M. Neurodevelopmental outcomes of preterm birth. New York: Cambridge University Press;2010.

21) Chiriboga CA, Kuban KC, Durkin M, Hinton V, Kuhn L, Sanocka $\mathrm{U}$, et al. Factors associated with microcephaly at school age in a very-low-birthweight population. Dev Med Child Neurol 2003; 45:796-801.

22) Brown L, Burns YR, Watter P, Gibbons KS, Gray PH. Motor performance, postural stability and behaviour of non-disabled extremely preterm or extremely low birth weight children at four to five years of age. Early Hum Dev 2015;91:309-315.

23) Pomella R, Baldino R, Cravero B. Cognitive, emotional and behavioral development of VLBW and ELBW preterm infants: diagnostic and therapeutic follow-up at preschool age. Minerva Pediatr 2013;65: 631-643.

24) Hughes MB, Shults J, McGrath J, Medoff-Cooper B. Temperament characteristics of premature infants in the first year of life. J Dev Behav Pediatr 2002;23:430-435.

25) Goldstein DJ, Bracey RJ. Temperament characteristics of toddlers born prematurely. Child Care Health Dev 1988;14:105-109.

26) Ham BJ, Choi MJ, Lee HJ, Kang RH, Lee MS. Reward dependence is related to norepinephrine transporter T-182C gene polymorphism in a Korean population. Psychiatr Genet 2005;15:145-147.

27) Garvey MJ, Noyes R Jr, Cook B, Blum N. Preliminary confirmation of the proposed link between reward-dependence traits and norepinephrine. Psychiatry Res 1996;65:61-64.

28) Gerra G, Zaimovic A, Timpano M, Zambelli U, Delsignore R, Brambilla F. Neuroendocrine correlates of temperamental traits in humans. Psychoneuroendocrinology 2000;25:479-496.

29) Pud D, Eisenberg E, Sprecher E, Rogowski Z, Yarnitsky D. The tridimensional personality theory and pain: harm avoidance and reward dependence traits correlate with pain perception in healthy volunteers. Eur J Pain 2004;8:31-38. 